\title{
The Effect of Cs-Correction on Filtered Imaging and Spectral Energy Resolution for a Post-Column Imaging Energy Filter
}

\author{
M.M.G. Barfels, M. Kundmann, C. Trevor, J.A. Hunt \\ Gatan Inc., 5933 Coronado Lane, Pleasanton, CA 94588
}

The emergence of commercially available Cs aberration corrected transmission electron microscopes [1] in combination with monochromators [2,3] raises the question how spherical aberration correction may affect the energy resolution of a high resolution sub-50 meV electron energy loss spectrometer [4]. Spherical aberrations of the objective lens are not only detrimental to spatial resolution, but can also significantly degrade the spectral energy resolution of an electron energy loss spectrum or limit the slit width used for energy-filtered diffraction imaging.

The filter's electron optical energy resolution is a function of 1.) filter aberrations and 2.) the optical coupling of the specimen with the spectrometer, called image-spectrum mixing. An energy filter can be coupled to the microscope optics either in diffraction mode or in image mode. A postcolumn filter focuses the projector lens crossover into an energy dispersed spectrum. In diffraction mode the projector crossover contains an image of the specimen. Conversely in image mode the projector crossover contains a diffraction pattern of the specimen.

Most electron energy loss spectroscopy (EELS) measurements, STEM spectrum imaging and energy filtered diffraction patterns are acquired in the image-coupled filter mode. In comparison to diffraction-spectrum mixing much less is known about image-spectrum mixing. The effect of diffraction-spectrum mixing has been shown for both in-column [5,6] and post-column filters $[7,8]$. It has also been mathematically presented in terms of transmissivity [9], which is the imaged area as a function of solid scattering angle that can be passed through the filter for a given energy resolution defined by the filter aberrations. Calculating the size of the image in the projector crossover using the approach in [9], then for a camera length of $15 \mathrm{~mm}$ and a probe size of $5 \mathrm{~nm}$ the image size in the spectrum of the post-column filter is only $0.08 \mathrm{eV}$. However, this analysis neglects the effect of spherical aberrations of the objective lens. Accounting for spherical aberrations, it can be shown that the size of the projector crossover in the spectrum at the disc of minimum confusion with an objective lens Cs of $1.1 \mathrm{~mm}$ and a $100 \mathrm{mrad}$ collection angle amounts to $9.3 \mathrm{eV}$. This is a much more realistic value of the projector crossover size in the spectrum of an image-coupled filtered mode. If most of the image magnification is obtained with the objective lens, the aberrations of all the other electron microscope lenses are expected to be far less in shaping the projector crossover waist. This condition may vary for different microscopes.

A post-column filter with partial $3^{\text {rd }}$ order aberration correction and a $5 \mathrm{~mm}$ entrance aperture was used to acquire an energy filtered CBED pattern of $\mathrm{Si}<111>$ at $200 \mathrm{kV}$ on a conventional TEM with a LaB6 emitter. The filter aberrations are shown in FIG 1. The non-isochromaticity is $1.5 \mathrm{eV}$. The Cs of the objective lens is $1.1 \mathrm{~mm}$. FIG 2 shows an energy filtered diffraction pattern, which is an image-coupled filter mode with an energy window of $+/-5 \mathrm{eV}$. Extracting the zero-loss shift from this pattern, a strong third order component can be seen (FIG 3). The circular shape of the aberration in FIG 3 can not be produced with the energy-dispersive magnet shape of the presented filter. Furthermore, the $10 \mathrm{eV}$ energy window supports the theoretical estimate of a $9.3 \mathrm{eV}$ effect of 
the objective lens spherical aberration in the spectrum for a $100 \mathrm{mrad}$ collection angle. At these large angles the size of the imaged specimen area of a convergent beam becomes negligible.

If smaller energy windows are desired for a given collection angle, then a filter with higher aberration correction is needed to allow for a greater aperture size. Larger filter entrance apertures reduce the magnification of the filter to the detector. This permits the use of a larger pre-filter camera length, reducing the effect of image-spectrum coupling for the same collection angle and consequently allowing for a smaller energy window for an energy filtered CBED image. Such a filter has been designed for monochromated TEMs [4].

A comparative study of energy filtered CBED imaging will be presented for three different systems with a $5 \mathrm{~mm}$ and an effective $5.4 \mathrm{~mm}$ and $6.5 \mathrm{~mm}$ filter aperture respectively. The latter two systems have full $3^{\text {rd }}$ order and partial $4^{\text {th }}$ order aberration correction. In addition, the effect of a lower Cs on the energy loss spectrum will be presented.

[1] Haider et al., Nature 392 (1998) 768

[2] Tiemeijer, P.C., Inst. Phys. Conf. Ser. No.161: section 5 (1999) 191

[3] Freitag et al., Ultramicroscopy 102 (2005) 209

[4] Brink et al., Ultramicroscopy 96 (2003) 367

[5] Crozier, P.A. Ultramicroscopy 58 (1995) 157

[6] Reimer, L. Energy-Filtering Transmission Electron Microscopy, Springer Vrlg Berlin(1995)

[7] Krivanek et al., Ultramicroscopy 59 (1995) 267

[8] Kothleitner and Hofer, Micron 34 (2003) 211

[9] Uhlemann et al., Ultramicroscopy 63 (1996) 161

\section{$1.5 \mathrm{eV}$}
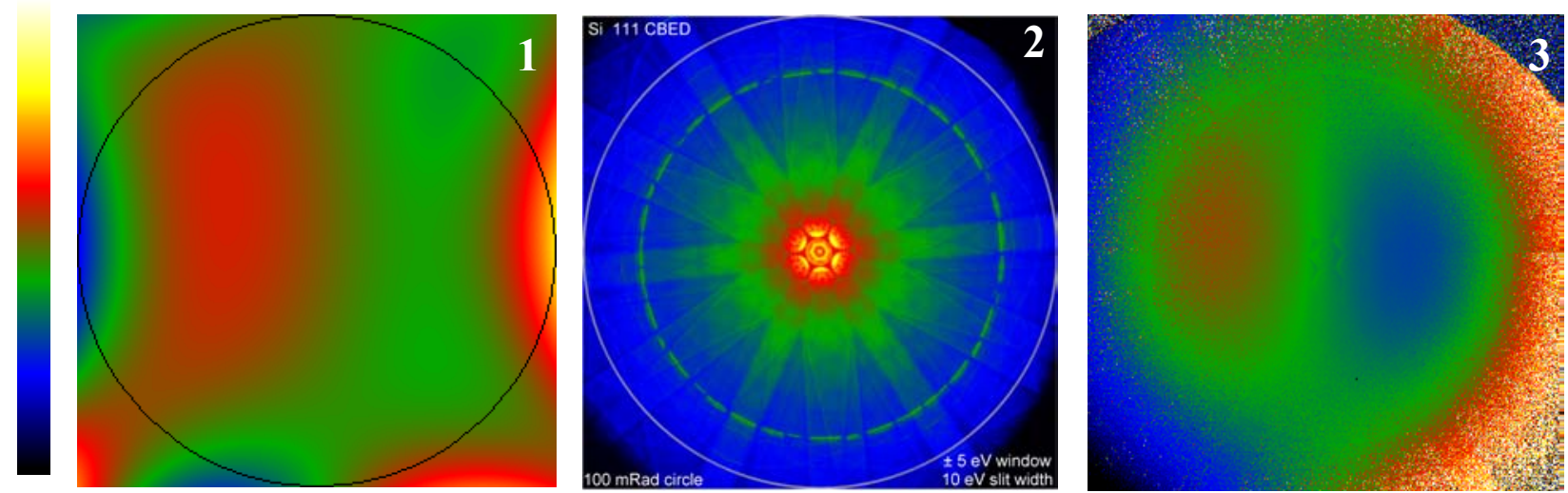

$0 \mathrm{eV}$

FIG 1: Non-isochromaticity of a full $2^{\text {nd }}$ order and partial $3^{\text {rd }}$ order corrected imaging energy filter. The maximum variation in energy across the detector as a result of filter aberrations is $1.3 \mathrm{eV}$.

FIG 2: Energy filtered CBED of $\mathrm{Si}<111>$ at $200 \mathrm{kV}$ with a $+/-5 \mathrm{eV}$ energy window. The outer circle represents a $100 \mathrm{mrad}$ collection angle.

FIG 3: The energy variation in the spectrum plane of the zero loss electrons for the CBED pattern shown in FIG.2. The cut-off in the upper right hand corner is from an aperture above the filter, possibly the differential pumping aperture. 